Archives

17 | 1996

Hommage à Bernard Lepetit

\title{
La gestion de l'eau potable en France
}

\section{Aramires França}

\section{(2) OpenEdition}

Journals

Édition électronique

URL : http://journals.openedition.org/ccrh/2598

DOI : $10.4000 /$ ccrh. 2598

ISSN : $1760-7906$

Éditeur

Centre de recherches historiques - EHESS

Édition imprimée

Date de publication : 4 octobre 1996

ISSN : 0990-9141

Référence électronique

Aramires França, "La gestion de l'eau potable en France », Les Cahiers du Centre de Recherches Historiques [En ligne], 17 | 1996, mis en ligne le 27 février 2009, consulté le 10 décembre 2020. URL http://journals.openedition.org/ccrh/2598; DOI : https://doi.org/10.4000/ccrh.2598

Ce document a été généré automatiquement le 10 décembre 2020.

Article L.111-1 du Code de la propriété intellectuelle. 


\title{
La gestion de l'eau potable en France
}

\author{
Aramires França
}

\section{Introduction}

1 Nous posons comme hypothèse initiale que les normes internationales récentes sur l'eau potable ont contribué à renforcer sensiblement le rôle des gestionnaires du service de la fourniture d'eau potable. La notion de l'aspect normatif en matière d'eau potable soulève des difficultés notamment pour ceux qui mettent en évidence la fonction régulatrice dévolue à l'administration. Les transformations de la structure du droit interne peuvent être la conséquence de l'appartenance de l'État à l'organisation internationale. On peut dire que le droit administratif est le champ le plus sensible aux transformations mises en œuvre dans la société.

2 Toutes les cultures antérieures à la nôtre ont trouvé l'art d'obtenir une eau agréable à boire. Cette consommation a une histoire avec ses limites, inévitables : il s'agit, en effet, d'une lutte étroitement liée au droit à la santé.

\section{Considérations méthodologiques}

3 Lorsqu'il s'agit de rechercher quel est le contenu, donc la signification, de la norme internationale en matière d'eau potable, on considère d'abord qu'elle est reconnue dans différents contextes nationaux, soit par une disposition constitutionnelle, soit par la législation ordinaire.

4 La stratégie élaborée pour répondre à la question de l'impact de la norme commence par l'hypothèse suivante: la lecture dogmatique du texte normatif est insuffisante comme méthode d'observation. L'idée est qu'il faut interpréter la norme à partir de la connaissance $d u$ contexte où elle s'est développée. La démarche de recherche est d'étudier d'abord le contexte international avant la norme de l'OMS pour l'eau de boisson; puis le contenu du texte normatif, les principes fondamentaux, les objectifs ; enfin, l'exemple d'un champ concret d'application, le cas français. 


\section{Antécédents}

Bien avant que la notion de contagion fut scientifiquement établie, les grandes épidémies - particulièrement de peste qui ravagèrent le bassin méditerranéen - en imposèrent l'idée au public autant qu'aux médecins ${ }^{1}$.

Les mesures de protection prises à ce moment-là étaient d'isoler les malades en les mettant en quarantaine, surtout ceux qui venaient des zones portuaires.

$C^{\prime}$ 'est surtout au moment du commencement, quand s'organisent et se multiplient les pèlerinages de La Mecque, comme à l'époque des Croisades, que le brassage des populations provoqué par ces événements entraîne la propagation des terribles maladies endémiques que sont la peste, le choléra et le typhus ${ }^{2}$.

En 1664, Londres a perdu un tiers de sa population dans une terrible épidémie de peste.

7 L'inoculation de la variole est pratiquée en Grande-Bretagne depuis les années 1720, et, au cours des deux décennies suivantes, cette méthode est déjà connue sur le continent européen, mais la vaccination apparait en 1800. Les sommités scientifiques ont été convoquées aux conférences internationales d'hygiène vers la moitié du XIX ${ }^{\mathrm{e}}$ siècle. Un nombre raisonnable de pays sont présents à ces conventions dont l'objectif recherché était une voie de coopération inter-étatique pour aboutir à la défense de la santé.

L'expérience avait montré que le recours aux méthodes traditionnelles des négociations bilatérales entre diplomates professionnels ne permettait pas de résoudre avec la rapidité voulue les problèmes techniques qui exigeaient d'être étudiés simultanément par de nombreuses nations: le moment était à la convocation de conférences internationales ayant pour objet de permettre aux nations d'aboutir à des accords sur de nombreuses questions de caractère non politique ${ }^{3}$.

$8 \mathrm{Au}$ début $\mathrm{du} \mathrm{xx}^{\mathrm{e}}$ siècle, un ensemble de dix conventions sanitaires à caractère international avait déjà discuté des problèmes endémiques qui menaçaient les nations, mais les résultats de ces rencontres scientifiques étaient de portée limitée.

\section{Le rôle des institutions sanitaires internationales}

9 En 1902 les républiques américaines ont créé le Bureau sanitaire panaméricain (BSP). En 1907, des délégations de la Belgique, du Brésil, de l'Égypte, de l'Espagne, des États-Unis, de la France, de la Grande-Bretagne, de l'Italie, des Pays-Bas, du Portugal, de la Russie et de la Suisse ont signé l'Arrangement de Rome instituant à Paris l'Office international d'hygiène publique (OIPH). Le nouvel organisme était sous l'autorité et le contrôle d'un comité composé des délégués, représentants techniques des gouvernements membres, et avait pour mission principale de communiquer aux États participants des informations de caractère général intéressant la santé publique et en particulier des renseignements sur les maladies transmissibles notamment la peste, le choléra et la fièvre jaune, ainsi que sur les mesures prises pour les combattre.

10 Le but principal de l'OIPH était de faire attention aux maladies transmissibles, mais elle a joué un rôle fondamental dans les domaines administratifs des États représentés. Il ne s'agissait pas d'un simple bureau international d'information, mais surtout d'un forum pour réfléchir aux grandes endémies.

11 Après la guerre de 14-18, le typhus ravageait l'Europe de l'Est et l'URSS. Ce dernier pays enregistrait plus de 1600000 cas et la Pologne, 200000 cas. En 1920, la Conférence 
internationale d'hygiène fut convoquée à la demande du Conseil de la SDN. Seulement cinq pays y étaient représentés : la France, la Grande-Bretagne, l'Italie, le Japon et les États-Unis. La recommandation principale fut la création, dans le cadre de la SDN, d'un organisme permanent d'hygiène au sein duquel devait rester l'OIPH. Les États-Unis ont refusé le rattachement de l'OIPH au nouvel organisme, qui ne fut créé qu'en 1923. Cette organisation fut admise comme bureau général consultatif d'hygiène au Comité du secrétariat de la SDN, qui va développer son action, à partir des commissions, sur le cancer, la lèpre, la standardisation biologique, l'habitation, l'enseignement de l'hygiène, l'hygiène rurale et les «maladies sociales »-c'est-à-dire les maladies résultant de la malnutrition.

\section{Les objectifs de l'OMS}

12 La constitution de l'Organisation mondiale de la santé a pour but d'«amener tous les peuples au niveau de santé le plus élevé possible» (Art.1). La politique générale de l'organisation est arrêtée par les représentants de tous les membres lors de la réunion annuelle de l'Assemblée mondiale de la santé (Art. 9-23). Son conseil exécutif est composé de trente délégués élus par l'assemblée, décidant à titre individuel, même s'ils sont désignés par leurs gouvernements (Art. 24-37).

13 Le monde est divisé, par l'OMS, en six régions (les arrangements régionaux). Chacune comprend un comité régional, auquel participent les membres de l'arrangement concerné, et un bureau régional, sous la charge du directeur régional, désigné par le conseil régional en accord avec le conseil exécutif, qui le nommera (Art. 40-45).

\section{Les aspects normatifs pour l'eau en France avant les normes de l'OMS}

L'analyse du contexte international qui aboutit à l'élaboration des normes internationales pour l'eau de boisson montre que la participation de la France fut décisive. Sa présence aux conférences internationales d'hygiène est remarquable. Il y avait une tradition réglementaire née des préoccupations sanitaires.

Face aux maladies, jamais autant qu'au XIX siècle la pureté de l'eau ne fut à ce point au cœur des débats, la thèse de Jean-Pierre Goubert nous l'indique ${ }^{4}$. Chez l'élite savante et politique, elle fait l'objet d'attentions et de soins rigoureux. Désormais, surtout à la fin du siècle, la connaissance scientifique de l'eau s'inscrit dans un vaste projet de transformation sociale et culturelle né des grandes utopies de l'époque.

\section{La loi de 1902 sur la santé publique}

Notre hypothèse sur cette loi est qu'elle peut être considérée comme le point de départ de l'interventionnisme d'État dans le domaine de l'eau. Avant cette loi, l'État français a réglé les conflits sur l'utilisation de l'eau dans le droit de propriété. La loi du 8 avril 1898 était le dispositif majeur dans le domaine de l'eau.

Au Congrès international d'hygiène à Paris, en 1900, 
F. Launay, ingénieur en chef de l'assainissement de Paris, demande la création d'un service de surveillance pour les eaux destinées à la capitale. Quelque temps après, le préfet de la Seine se range à cet avis et a créé le service souhaité 5 .

\section{Le décret de 1935 : la protection des ressources en eau souterraine} moins limitées en quantité et moins vulnérables aux pollutions. Le décret de 1935 protégea, dans un premier temps, les ressources en eau souterraine de la région parisienne en soumettant à l'autorisation les puits ou sondages de plus de 80 mètres de profondeur.

Aux zones initialement concernées par cette règle, d'autres se sont par la suite ajoutées, limitant les prélèvements en profondeur ou en débit ${ }^{6}$.

\section{Les normes techniques pour rendre une eau pure (1929-1954)}

19 Il faut attendre la circulaire du 12 août 1929 pour avoir des indications relatives aux procédés de correction et de stérilisation.

Cette philosophie basée sur des procédés domina jusqu'en 1954, date à laquelle le nombre des normes augmente considérablement. En effet, une instruction qui accompagne la circulaire du 24 novembre 1954 traduit les progrès des méthodes d'analyse en termes de qualité7.

\section{L'évolution technique et les effets des guerres}

M. Guillaume, dans Quarante Ans de politique de l'eau en France, admet que les principes de base qui régissent les distributions d'eau sont invariables. L'utilisation de canaux, aqueducs, machines élévatoires et tuyaux sont les exemples indiqués, qui sont apparus au $\mathrm{XVII}^{\mathrm{e}}$ siècle. L'évolution des techniques portant sur le traitement des eaux pour en améliorer la qualité est, au contraire, récente. M. Loriferne remarque que la situation en France après 1944 a conduit le gouvernement à centraliser les sous-systèmes économique et politique. Les autorités locales, c'est-à-dire les conseils municipaux pour les communes et généraux pour les départements, gardèrent leur autonomie. "Mais toutes les collectivités fonctionnaient avec une très forte tutelle de l'État $»^{8}$.

La politique de l'État français est alors celle de la planification. Cette adaptation était urgente car les productions nationales n'accompagnaient pas la croissance des échanges internationaux, aussi la France, confrontée à une compétition économique qui s'intensifie, signe le traité de Rome, en 1957, qui crée la Communauté économique européenne (CEE).

L'alignement de la France sur les normes internationales de production et de consommation est net; ainsi que dans le domaine technique, le pays a adopté les normes internationales pour l'eau de boisson, instituées par l'OMS. L'année suivante, une Commission de l'eau fut créée.

\section{L'impact de la norme en France}

L'hypothèse que nous essayons de montrer est que les normes internationales pour l'eau de boisson, imposées par l'OMS en 1958, ont produit des effets définitifs sur l'ensemble de 
l'action administrative française en matière d'eau potable. Avant 1958, un ordre juridiquement établi a imposé des transformations et même la fameuse loi de 1964, qui se présente dans la majorité des ouvrages comme une politique nouvelle et progressive de gestion de l'eau du pays, n'a pas changé les orientations principales imposées par l'ordonnance $\mathrm{n}^{\circ}$ 58-1265 du 20 décembre 1958. En France, l'autorité des traités internationaux est «supérieure à celle des lois même postérieures, et c'est le traité que l'administration devra appliquer si la loi lui est contraire $»^{9}$.

\section{Les textes normatifs en matière d'eau potable après les normes de I'OMS : l'ordonnance n 58-1265 du 20 décembre 1958}

24 À la suite de la publication des normes internationales de l'eau de boisson, on vit l'État organiser la même année l'administration des distributions publiques, privées et communes en matière d'eau potable :

Il s'agit d'une décision prise par le gouvernement en vertu de l'habilitation délibérée en Conseil des ministres après avis du Conseil d'État, une ancienne pratique de loi mise en élaboration dans le cadre des régimes exceptionnels ${ }^{10}$.

Les aspects concernant le mode de distribution des eaux potables sont définis par ce dispositif valable encore aujourd'hui et inscrit au chapitre III du Code de la santé publique. Par rapport aux distributions privées, celles-ci doivent être soumises à l'autorisation du préfet, qui peut la suspendre ou la retirer (Art. L. 24).

\section{Vers la nouvelle politique de l'eau}

Pour M. Picard, l'ensemble de la législation et de la réglementation était insuffisant en ce qui concerne le régime des eaux, le droit d'usage de l'eau et sa répartition comme ce qui concerne la pollution :

La législation était éparse et sectorielle, car la pollution n'a pas été considérée comme un phénomène unique, susceptible de modes de traitement communs ${ }^{11}$.

\section{La loi de 1964 et la gestion des services de l'eau}

D'après M. Gleizes, il s'agit d'une

[...] loi-cadre qui a défini les objectifs et créé des moyens d'action en se référant à des principes :

- l'unité de la ressource en eau dans le cadre des bassins hydrographiques,

- la conciliation des intérêts des usagers publics et privés.

Elle laisse aux textes réglementaires, le soin de préciser les moyens à mettre en œuvre ${ }^{12}$

En ce qui concerne l'eau potable, la loi de 1964 complète l'ordonnance de 1958 quand elle institue les paramètres de protection des captages. Deux aspects de cette loi sont incorporés au Code de santé publique: la protection de la qualité des eaux ${ }^{13}$ et les indemnités ${ }^{14}$.

En France, les bases juridiques des normes techniques sont évidentes

[...] parce qu'elles sont souvent complétées par des circulaires, instructions techniques émanant d'un ministère à l'intention de ses agents. Ces documents ne relèvent pas du domaine législatif ou réglementaire et ne sont donc pas opposables aux tiers, mais servent de guide pour l'élaboration des autorisations 
administratives et des documents contractuels. En cas de conflit porté devant les tribunaux, les juridictions utilisent fréquemment ces instructions pour éclairer leur jugement ${ }^{15}$.

\section{Le contexte normatif régional : les directives de la CEE}

Nous avons montré, au début de notre étude, la formation de plusieurs organisations à caractère international, créées dès le début du siècle, en matière de défense de santé publique. L'existence de ce genre d'organisation a géré l'apparition des normes émanant d'un organisme international qui sont venues compléter l'ordre juridique existant au niveau de chaque État, qui les intègre. confédération et la constitution d'États. Mais ce n'est pas la même chose de parler de la coopération entre États et de parler de l'intégration qui peut être produite entre États. La coopération traduit la quête à partir des confrontations et discussions permanentes, des accords librement consentis et l'intégration délimite une nature juridique nouvelle parce qu'elle suppose une structure économique et politique intégrée.

\section{Les textes normatifs communautaires en matière d'eau potable}

- a) La directive $n^{\circ} 75 / 440 /$ CEE du 16 juin $1975^{16}$. Ce dispositif, concernant la qualité requise des eaux superficielles destinées à la production d'eau alimentaire dans les États membres, fait partie du programme d'action des Communautés européennes en matière d'environnement, qui a prévu les objectifs de qualité et les exigences qu'un milieu doit satisfaire. Il définit les paramètres valables pour l'eau, y compris les eaux douces superficielles utilisées ou destinées à être utilisées à la production alimentaire. Les dispositions législatives, réglementaires et administratives nécessaires à la conformité de cette directive doivent être mises en vigueur dans un délai de deux ans (Art. 10).

- b) La directive $n^{\circ} 79 / 869 /$ CEE du 9 octobre 1969. Ce dispositif, relatif aux méthodes de mesure et à la fréquence des échantillonnages et de l'analyse des eaux superficielles destinées à la production d'eau alimentaire dans les États membres, représente un complément qui considère le progrès technique et scientifique dans le domaine de l'eau potable. Il concerne la désignation d'un principe de mesure ou la description d'un processus opératoire qui permettent la détermination des paramètres, la limite et la détection, la précision, l'exactitude, c'est-à-dire la différence entre la valeur réelle du paramètre examiné et la valeur moyenne expérimentale obtenue (Art. 2).

- c) La directive $n^{\circ} 80 / 778 / C E E$ du 15 juillet 1980. Cette directive, relative à la qualité des eaux destinées à la consommation humaine, définit toutes les eaux utilisées à cette fin, en l'état ou après traitement, quelle que soit leur origine : qu'il s'agisse d'eaux livrées à la consommation ou d'eaux utilisées dans une entreprise alimentaire, ou encore de mise sur le marché de substances ou de produits destinés à être consommés par l'homme et affectant la salubrité de la denrée alimentaire finale (Art. 2). Les eaux minérales et les eaux médicinales sont exclues de cette directive (Art. 4).

la même manière que lors des publications de l'OMS en 1958.

Malgré la pression européenne, la France tarde à se mettre à jour et la Commission

de Bruxelles le lui rappelle fermement en $1987^{17}$. 
À cette date, le pays se contente de respecter la dernière réglementation de 1961. Il faut attendre le 3 janvier 1989 pour que la France édicte son décret d'application de la CEE de $1980^{18}$.

\section{Conclusion}

Tout au long de la démarche, nous essayons de montrer, à partir d'une approche normative internationale, les effets produits au niveau de l'organisation interne, en France, des services publics chargés de la fourniture d'eau potable.

L'après-guerre dut marquée par l'intérêt mondial pour l'élaboration d'une loi sur l'eau. Très souvent le phénomène de l'urbanisation est avancé comme hypothèse explicative, de même les préoccupations sanitaires et les avancements techniques.

L'expert en technologie de pointe dans la production d'eau est fier de dire que «quelle que soit la qualité de l'eau, on sait la traiter ». Bien sûr, mais les pouvoirs chargés du service d'intérêt public en matière d'eau se doit de lui demander quel sera le prix de cette eau et qui en paiera le coût?

Le désir de réorganiser un système normatif qui prendrait en compte les dispositions relatives tant aux produits pour les traitements d'eau potable qu'aux procédés et normes de résultats dans un cadre techniquement complexe, laisse le citoyen commun, autrement dit le consommateur, tout à fait absent des sphères de décision.

L'impact d'une norme n'est pas toujours évident parce qu'il faut attendre la mise ne application pour vérifier les objectifs placés au début et les transformations perçues au niveau du champ d'application.

L'analyse des aspects normatifs nous donne une dimension interprétative assez riche du point de vue méthodologique. La tentative de saisir le sous-système " droit » au sein d'un pays ne pose pas de problème théoriquement insoluble, mais l'expérience nous a enseigné d'avancer vers un approfondissement de la problématique que nous avons posée au début.

\section{Sources}

Code de l'environnement, Paris, Dalloz, $2^{\mathrm{e}}$ édition, 1984.

Code de la santé publique, Paris, Dalloz, 1989.

Directives de qualité pour l'eau de boisson, 3 vol., Genève, OMS, 1985.

" Hygiène alimentaire, eaux destinées à la consommation humaine ", Paris, Journal officiel de la République française, brochure $\mathrm{n}^{\circ} 1629,1991$.

Normes internationales pour l'eau de boissons, $3^{\mathrm{e}}$ édition, Genève, OMS, 1972.

Traités instituant les Communautés européennes, Luxembourg, éditions abrégées, 1987. 


\section{NOTES}

1. R. Dujarric de la Rivierie, Prophylaxie nationale et internationale des maladies épidémiques, Paris, Flammarion, 1948, p. 173.

2. Antoine Zarb, Les Institutions spécialisées du système des Nations unies et leurs membres, Paris, Pedonne, 1980, p. 24.

3. Marcolino Candau, Deuxième Décennie de l'OMS, p. 5.

4. Jean-Pierre Goubert, La Conquête de l'eau, Paris, Laffont, 1986, p. 97.

5. Ibid., p. 100.

6. M.-G. Fontenier, L'Alimentation en eau potable en Basse-Normandie, mémoire de DEA de géographie, université de Caen, 1989, p. 17.

7. B. Barraque et al., Normes et choix techniques dans les domaines de l'eau, de l'assainissement et des déchets : collectivités locales, État, ingénieurs, rapport pour le CNRS-PIREN, 1990.

8. Hubert Loriferne (dir.), Quarante Ans de politique de l'eau en France, Paris, Economica, 1987, p. 50.

9. Jean Rivero, Droit administratif, Paris, Dalloz, 1990, p. 65.

10. Ibid., p. 82.

11. Hubert Loriferne, op. cit., p. 61.

12. Ibid., p. 67.

13. Art. 7 de la loi $n^{\circ} 64-1245$ incorporé comme art. L. 20 au Code de la santé publique.

14. Art. 8 de la loi $n^{\circ} 64-1245$ devenu art L. 20-1 du Code de la santé publique.

15. P. Boutin, R. Seux, «Les aspects socio-juridiques de la normalisation en matière d'eau potable ", revue La Technique de l'eau et de l'assainissement, $n^{\circ}$ 435-436, mars-avril 1983, p. 10.

16. Publié au J.O. $\mathrm{n}^{\circ} 112$ du 20 décembre 1973.

17. B. Barraque, op. cit., p. 43.

18. Décret $\mathrm{n}^{\circ}$ 89-3 du 3 janvier 1989 (Art. 4).

\section{AUTEUR}

\section{ARAMIRES FRANÇA}

Architecte, université fédérale de Rio Grande do Norte (Brésil), 1978. Mestrado en aménagement urbain, université de Brasília, 1983.

EHESS, sous la direction de Bernard Lepetit: DEA « Territoires urbains », 1993. Doctorante. Chercheur à la fondation José Augusto, Natal (Rio Grande do Norte, Brésil). 